\title{
ON TRANSIENT THERMAL STRESSES IN AN INFINITE THIN PLATE
}

\author{
by W. DERSKI \\ (Received 30th June 1960)
}

1. THE presence of a non-steady state of temperature in an elastic solid gives rise to an additional term in the generalised Hooke's Law connecting the stress and strain tensors and terms involving the time rate of change of the dilatation. This time-dependent dilatation may produce so-called thermoelastic stress waves. The present note is concerned with the effects produced by these additional terms in a simple situation, in which the elastic solid is regarded as a thin plate of infinite extent. The distribution of temperature in the plate is produced by a point heat source of Dirac type.

2. The temperature field is determined by the equation

$$
\Delta T-\frac{1}{\kappa} \frac{\partial T}{\partial t}=-\frac{Q}{\kappa} \frac{\delta(r)}{2 \pi r} \delta(t)
$$

where, in cylindrical coordinates,

$$
\Delta=\frac{\partial^{2}}{\partial r^{2}}+\frac{1}{r} \frac{\partial}{\partial r}
$$

The solution has to satisfy the initial condition $\left.T(r, t)\right|_{t=0}=0$ and the temperature, when $r \rightarrow \infty$, must tend to zero. Such a solution is well known [see, for instance (2), (3), (4)], and has the form

$$
T(r, t)=\eta(t) \frac{Q}{4 \pi \kappa t} \exp \left(-\frac{r^{2}}{4 \kappa t}\right)
$$

The equations of thermo-elasticity reduce in our case to one equation in the displacement $u$ [see (3)]:

$$
\frac{\partial^{2} u}{\partial r^{2}}+\frac{1}{r} \frac{\partial u}{\partial r}-\frac{u}{r^{2}}-\sigma^{2} \frac{\partial^{2} u}{\partial t^{2}}=s \frac{\partial T}{\partial r}
$$

where $\sigma^{2}=\frac{1}{c^{2}}, s=(1+v) \alpha_{r}$.

The solution of the equation (3) has to satisfy the initial conditions

$$
\left.u(r, t)\right|_{t=0}=0 \text { and }\left.\frac{\partial u}{\partial t}\right|_{t=0}=0
$$

and two boundary conditions

E.M.S.-E.

$$
\left.u(r, t)\right|_{r=0}=0 \text { and } u(r, t)<\infty \text { when } r \rightarrow \infty .
$$


The initial conditions (4) enable us to apply the Laplace transform defined by the following integral

$$
u_{L}=\int_{0}^{\infty} u(r, t) e^{-p t} d t
$$

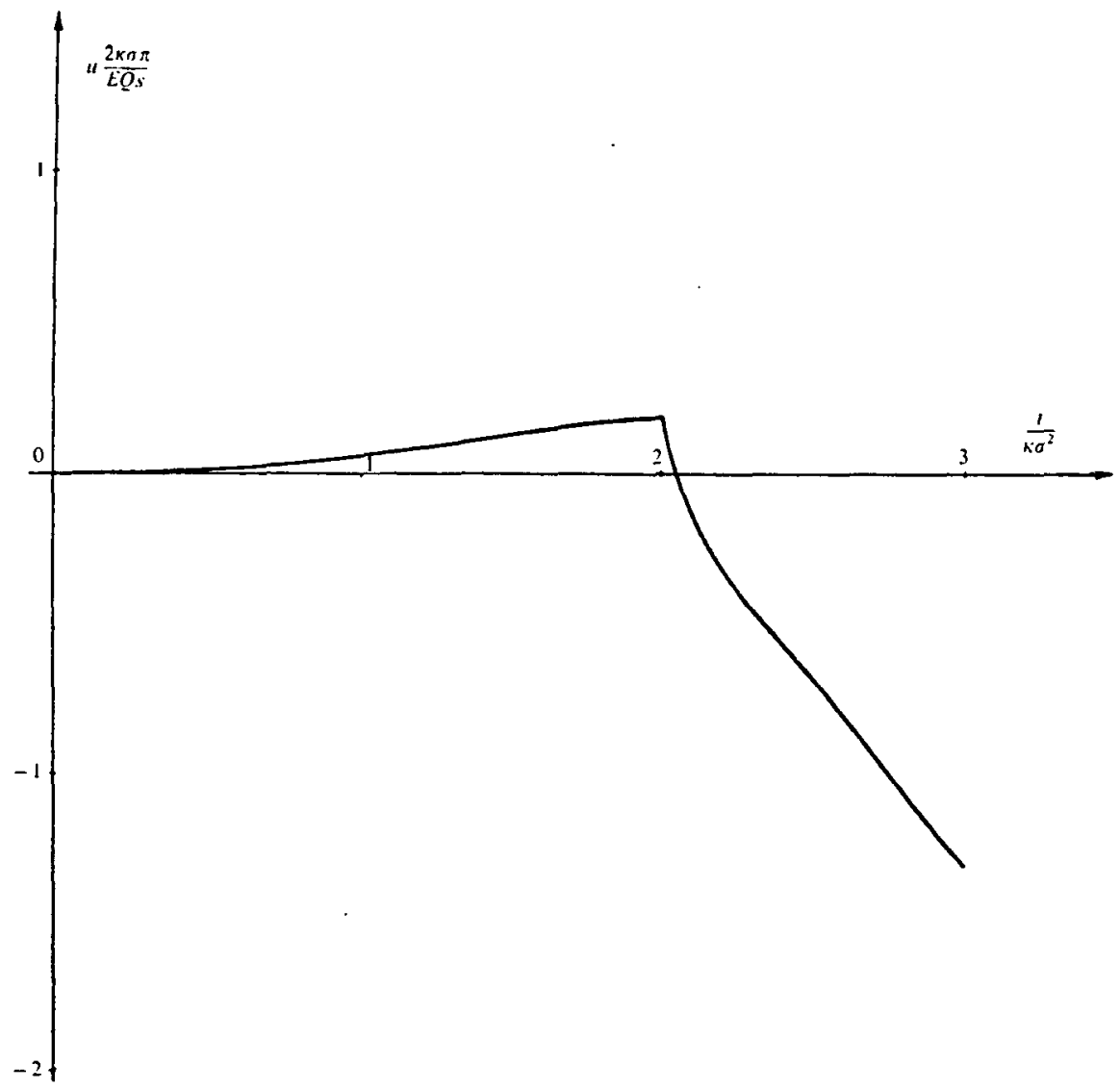

FIG. 1.-Displacement represented as a function of time for $\frac{r}{\kappa \sigma}=2$.

Applying the Laplace transform to both sides of the equation (3), we find (5)

$$
\frac{d^{2} u_{L}}{d r^{2}}+\frac{1}{r} \frac{d u_{L}}{d r}-\left(\frac{1}{r^{2}}+\sigma^{2} p^{2}\right) u_{L}=s \frac{d T}{d r}
$$

Performing the Hankel transform, defined by the integral

$$
u_{L H}=\int_{0}^{\infty} r u_{L} J_{1}(\alpha r) d r
$$


and taking into account the formula (2), we get the result in the form

$$
u_{L H}=\frac{s \alpha Q}{2 \pi \kappa\left(\alpha^{2}+q^{2}\right)\left(\alpha^{2}+\sigma^{2} p^{2}\right)} ; \quad q^{2}=\frac{p}{\kappa} .
$$

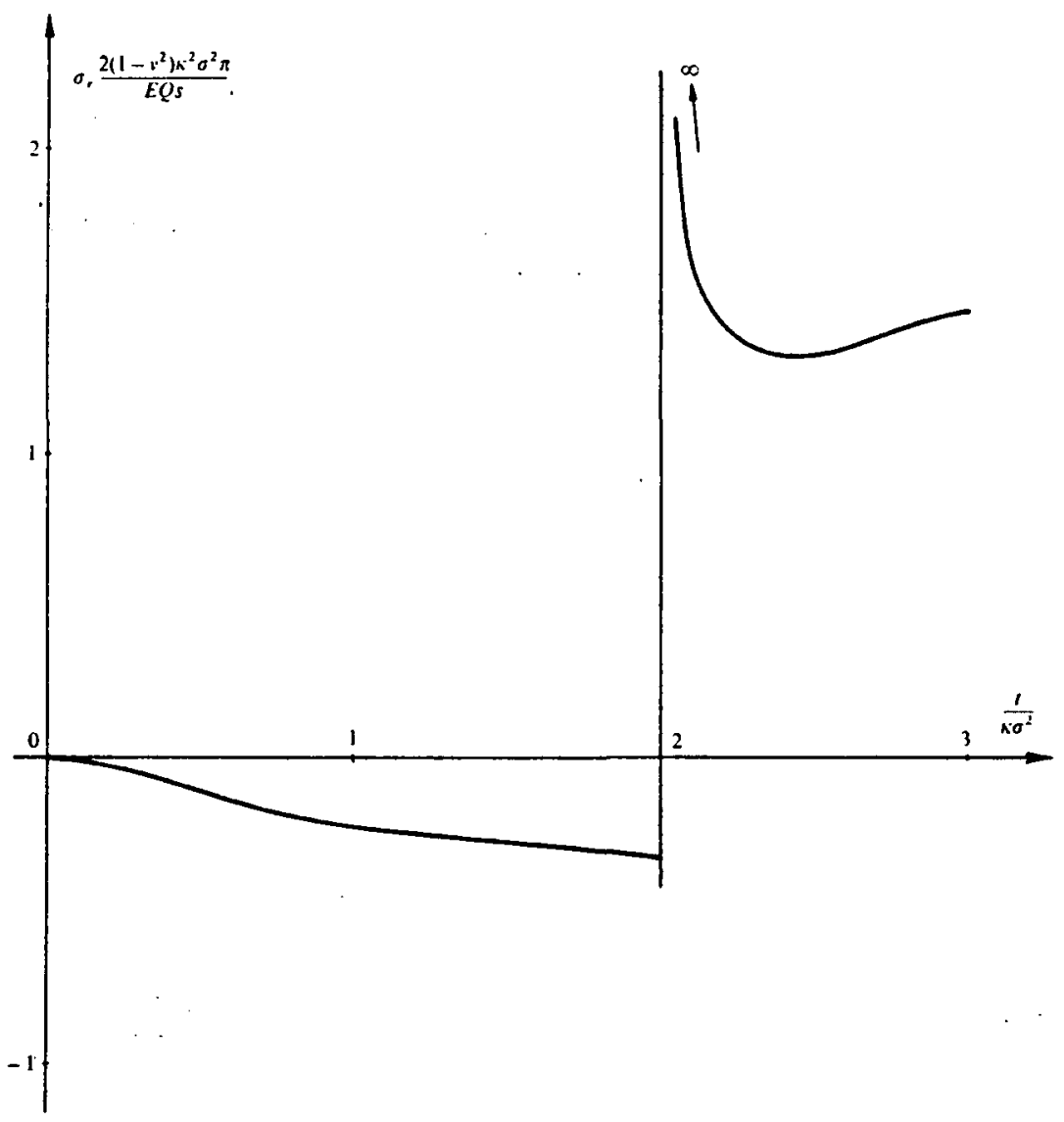

FIG. 2.-Stress represented as a function of time for $\frac{r}{\kappa \sigma}=2$.

The inverse Hankel transform leads to the following integrals

$$
u_{L}=\frac{s Q}{2 \pi p\left(\sigma^{2} \kappa p-1\right)}\left\{\int_{0}^{\infty} \frac{\alpha^{2} J_{1}(\alpha r)}{\alpha^{2}+q^{2}} d \alpha-\int_{0}^{\infty} \frac{\alpha^{2} J_{1}(\alpha r)}{\alpha^{2}+\sigma^{2} p^{2}} d \alpha\right\}
$$

Evaluating these integrals by known relations for modified Bessel functions of the second kind (6), we can write this formula in the form

$$
u_{L}=\frac{s Q}{2 \pi p\left(\kappa \sigma^{2} p-1\right)}\left[\sigma p K_{1}(\sigma p r)-q K_{1}(q r)\right] \text {. }
$$


Taking into account the convolution theorem, the inverse Laplace transform (1) gives the following form to the solution

$$
\begin{aligned}
u & =\frac{s Q}{2 \pi \kappa \sigma^{2} r}\left\{\eta(t) \exp \left(-\frac{t}{\kappa \sigma^{2}}\right) \int_{0}^{t} \exp \left(-\frac{r^{2}}{4 \kappa \xi}+\frac{\xi}{\kappa \sigma^{2}}\right) d \xi\right. \\
& \left.-\eta(t-\sigma r)\left[\sqrt{ }\left(t^{2}-\sigma^{2} r^{2}\right)+\frac{1}{\kappa \sigma^{2}} \exp \left(-\frac{t}{\kappa \sigma^{2}}\right) \int_{\delta r}^{t} \sqrt{ }\left(\xi^{2}-\sigma^{2} r\right) \exp \left(\frac{\xi}{\kappa \sigma^{2}}\right) d \xi\right]\right\}
\end{aligned}
$$

The thermal stresses associated with the displacement (9), are obtained by substitution of (9) and (2) into well-known relations

$$
\begin{aligned}
& \sigma_{r}=\frac{E}{1-v^{2}}\left(\frac{\partial u}{\partial r}+v \frac{u}{r}\right)-\frac{E \alpha_{T}}{1+v} T \\
& \sigma_{\vartheta}=\frac{E}{1-v^{2}}\left(\frac{u}{r}+v \frac{\partial u}{\partial r}\right)-\frac{E \alpha_{T}}{1+v} T
\end{aligned}
$$

and the results become:

$$
\begin{aligned}
& \sigma_{r}=-\frac{E Q s}{2 \pi\left(1-v^{2}\right)}\left\{\eta ( t ) \left[\frac{(1-v)}{\kappa \sigma^{2} r^{2}} \exp \left(-\frac{t}{\kappa \sigma^{2}}\right) \int_{0}^{t} \exp \left(-\frac{r^{2}}{4 \kappa \xi}+\frac{\xi}{\kappa \sigma^{2}}\right) d \xi\right.\right. \\
& \left.+\frac{1}{\kappa \sigma^{2}} \exp \left(-\frac{t}{\kappa \sigma^{2}}\right) \times \int_{0}^{t} \frac{1}{2 \xi} \exp \left(-\frac{r^{2}}{4 \kappa \xi}+\frac{\xi}{\kappa \sigma^{2}}\right) d \xi+\frac{(1-v)}{2 \kappa(1+v) t} \exp \left(-\frac{r}{4 \kappa t}\right)\right] \\
& -\eta(t-\sigma r)\left[\frac{(1-v) \sqrt{ }\left(t^{2}-\sigma^{2} r^{2}\right)}{\kappa^{2} \sigma^{4} r^{2}}+\frac{(1-v)}{\kappa \sigma^{2} r^{2}} \exp \left(-\frac{t}{\kappa \sigma^{2}}\right) \int_{\delta r}^{t} \sqrt{ }\left(\xi^{2}-\sigma^{2} r^{2}\right)\right. \\
& \left.\left.\times \exp \left(\frac{\xi}{\kappa \sigma^{2}}\right) d \xi+\frac{1}{\kappa \sqrt{ }\left(t^{2}-\sigma^{2} r^{2}\right)}+\frac{1}{\kappa^{2} \sigma^{2}} \exp \left(-\frac{t}{\kappa \sigma^{2}}\right) \int_{\sigma r}^{t} \frac{\exp \left(\frac{\xi}{\kappa \sigma^{2}}\right)}{\sqrt{\left(\xi^{2}-\sigma^{2} r^{2}\right)}} d \xi\right]\right\}, \\
& \sigma_{\vartheta}=\frac{E Q s}{2 \pi\left(1-v^{2}\right)}\left\{\eta ( t ) \left[\frac{(1-v)}{\kappa \sigma^{2} r^{2}} \exp \left(-\frac{t}{\kappa \sigma^{2}}\right) \int_{0}^{t} \exp \left(-\frac{r^{2}}{4 \kappa \xi}+\frac{\xi}{\kappa \sigma^{2}}\right) d \xi\right.\right. \\
& \left.-\frac{v}{\kappa \sigma^{2}} \exp \left(-\frac{t}{\kappa \sigma^{2}}\right) \int_{0}^{t} \frac{1}{2 \xi} \exp \left(-\frac{r^{2}}{4 \kappa \xi}+\frac{\xi}{\kappa \sigma^{2}}\right) d \xi-\frac{(1-v)}{2 \kappa(1+v) t} \exp \left(-\frac{r^{2}}{4 \kappa t}\right)\right] \\
& -\eta(t-\sigma r)\left[\frac{(1-v) \sqrt{ }\left(t^{2}-\sigma^{2} r^{2}\right)}{\kappa \sigma^{2} r^{2}}+\frac{(1-v)}{\kappa^{2} \sigma^{4} r^{2}} \exp \left(-\frac{t}{\kappa \sigma^{2}}\right) \int_{\sigma}^{t} \sqrt{ }\left(\xi^{2}-\sigma^{2} r^{2}\right) \exp \left(\frac{\xi}{\kappa \sigma^{2}}\right) d \xi\right. \\
& \left.\left.-\frac{v}{\kappa \sqrt{ }\left(t^{2}-\sigma^{2} r^{2}\right)}-\frac{v}{\kappa^{2} \sigma^{2}} \exp \left(-\frac{t}{\kappa \sigma^{2}}\right) \int_{\sigma}^{t} \frac{\exp \left(\frac{\xi}{\kappa \sigma^{2}}\right)}{\sqrt{\left(\xi^{2}-\sigma^{2} r^{2}\right)}} d \xi\right]\right\}
\end{aligned}
$$


We turn, finally, to the discussion of certain numerical results based on the dynamic solution to the transient thermoelastic problem treated in this paper. Our main objective is to determine the character of the results. The space and time dependence of the temperature field (2), as well as of the quasi-static displacement and stresses, were discussed extensively by E. Melan (4) and the corresponding diagrams need not be reproduced here.

Our graphs refer to the constant radius $\frac{r}{\kappa \sigma}=2$ and depend only on $t$. The dynamic solution involves also Poisson's ratio $v$, which is taken to have the value $0 \cdot 25$.

Fig. 2 shows, that in the case of a perfect elastic body, the stresses exhibit infinite jump-discontinuities at the wave-front.

\section{REFERENCES}

(1) A. ERdelyi, Editor, Tables of Integral Transforms, Vol. 1 (McGraw-Hill, 1954).

(2) G. EAson and I. N. SNeddon, The dynamic stresses produced in elastic body by uneven heating, Proc. Roy. Soc. Edin. A, 65, 143.

(3) W. NowACKI, Zagadnienia Termosprę̇ystosci (PWN, Warszawa, 1960).

(4) E. MeLAN, Spannungen infolge nichtstationärer Temperaturfelder (Österreich. Ing.-Archiv, 1955).

(5) I. N. SNeddon, Fourier Transforms (McGraw-Hill, 1951).

(6) G. N. Watson, A Treatise on the Theory of Bessel Functions (Cambridge, 1922).

Department of Mechanics of Continuous Media

Polish ACADEMY of SCIENCES 